\title{
Structure écologique et production fruitière de Haematostaphis barteri Hook. F en la zone sahélienne du Cameroun
}

\author{
Hamawa Yougouda1 ${ }^{1,}$, Balna Jules ${ }^{2}$, Souare Konsala ${ }^{3}$ \\ ${ }^{1}$ Département d'Agriculture, Élevage et Produits Dérivés, École Nationale Supérieure Polytechnique, Université de \\ Maroua, B. P. 46 Maroua, Cameroun, \\ 2 Institut de Recherche Agricole pour le Développement (IRAD), « Section Forêt », B.P 33 Maroua, Cameroun ; \\ ${ }^{3}$ Département des Sciences Biologiques, Faculté des Sciences, Université de Maroua ; B. P 814 Maroua, \\ Cameroun ; \\ *Auteur correspondant : hamawayougouda@yahoo.fr
}

Original submitted in on $10^{\text {th }}$ July 2018. Published online at www.m.elewa.org on $31^{\text {st }}$ October 2018 https://dx.doi.org/10.4314/jab.v130i1.10

\section{RESUME}

Objectif : évaluer la structure écologique et la production de fruits de Haematostaphis barteri pour fournir les informations nécessaires à une meilleure gestion et conservation de l'espèce au Cameroun.

Méthodologie et Résultats: Dans quatre sites, 48 parcelles d'un hectare ont servi pour des inventaires forestiers de Haematostaphis barteri et 120 arbres l'ont été pour quantifier la production. La distribution de diamètre montre une courbe en $\mathrm{J}$ renversé, caractéristique des populations jeunes. La densité varie de 13 à 17 individus/ha. La surface terrière varie de 0,28 à $0,49 \mathrm{~m}^{2} / \mathrm{ha}$, le diamètre varie de 17,31 à $20,57 \mathrm{~cm}$. Le nombre de fruits par panicule et par arbre varie de 15 à 28 et de 2346 à 7877 respectivement. L'analyse des paramètres dendrométriques et de production montre une différence significative $(P<0,001)$ entre populations.

Conclusion et Application des résultats: L'environnement est le facteur qui distingue le mieux les différentes populations et explique mieux les variations observées entre elles en ce qui concerne leurs caractéristiques structurelles et de production. Cependant il serait nécessaire de ressortir l'impact de la surexploitation sur la distribution et la production dans les différents sites.

Mots-clés: Structure écologique; Haematostaphis barteri; production fruitière; Zone sahélienne, Cameroun.

\section{INTRODUCTION}

Les forêts tropicales sont connues comme les écosystèmes les plus importants dans le monde en termes d'abondance et de diversité (Nguegim et al., 2010). Cette diversité joue un rôle important dans les écosystèmes des pays en voie de développement. Ils fournissent de nombreuses ressources naturelles (Sourou et al., 2016b) dont leur importance est particulièrement vitale pour les communautés locales des zones sahéliennes d'Afrique où ils sont utilisés comme combustible et source d'alimentation humaine et animale, tout en étant un élément régulateur des conditions agroclimatiques. Au Cameroun, beaucoup de Produits Forestiers Non Ligneux (PFNL) sont intéressants 
pour l'alimentation humaine et animale face aux besoins croissants qui découlent de la pression démographique. Malheureusement, ces espèces agroforestières sont menacées à cause de la dégradation continue des ressources naturelles (Wong et al., 2001). Haematosotaphis barteri est l'une de ces espèces alimentaires qui fournissent à la communauté locale une grande utilité traditionnelle à l'échelle régionale. Cependant, contrairement à certaines espèces telles que Parkia biglobosa, Vitellaria paradoxa, Adansonia digitata, Balanites aegyptiaca qui sont identifiées comme des espèces prioritaires dans la zone sahélienne du Cameroun (Fondoun, 2001), peu d'attention particulière a été accordée à Haematostaphis barteri, qui est malheureusement une espèce surexploitée et en danger (Adomou, 2005 ; Garnier, 2006). En effet ses différentes parties (feuilles, fruits, écorce, racines et bois) sont utilisées dans les domaines de la pharmacopée, l'alimentation et même les domaines rituels. L'usage excessif de ces différentes parties de l'espèce et la restriction de son habitat font d'elle une espèce en danger (Gautier et al., 2002). La maîtrise de la distribution de la diversité végétale

\section{MATERIEL ET METHODES}

Site d'étude: Ce travail a été conduit en zone sahélienne du Cameroun située entre $10,29181^{\circ}$ et $10,95590^{\circ}$ de Latitude Nord et $13,61175^{\circ}$ et $14,93973^{\circ}$ de Longitude Est. Le relief est constitué de plaines autour des Monts Mandara et un massif à la frontière avec le Nigeria. Ainsi, quatre villages (Pété, Mindif, Moutourwa et Kalfou) ont été retenus pour l'étude (Figure 1). L'altitude moyenne est de $1000 \mathrm{~m}$ dans les zones montagneuses et environ $300 \mathrm{~m}$ dans les plaines (Yengue et Callot, 2002). Le climat est du type Sahélien et les facteurs corollaires sont énormes pour la conservation et la gestion des écosystèmes. II est donc nécessaire que des études et des programmes appropriés soient menés pour améliorer la conservation et la gestion durable de Haematostaphis barteri au Cameroun. En plus du fait de l'importance écologique et socioéconomique de cette espèce, la connaissance de sa production en fruits serait utile pour contribuer à l'amélioration des conditions de vie de la communauté locale de la zone sahélienne du Cameroun puisque la vente de ses fruits peut rapporter un revenu substantiel à la communauté locale. En outre, la gestion durable de ressources naturelles renouvelables nécessite une analyse et une maîtrise des interactions multiples entre les facteurs humains, environnementaux et le complexe fonctionnement de l'écosystème savanicole. La présente étude vise donc à évaluer la structure écologique et la production de fruits de Haematostaphis barteri dans la zone sahélienne du Cameroun afin de fournir des informations nécessaires pour une meilleure gestion et conservation des populations de l'espèce.

avec deux saisons : une longue saison sèche allant de novembre à juin et une courte saison des pluies de juillet à octobre avec $800 \mathrm{~mm} / \mathrm{an}$ (Suchel, 1987). En plein saison sèche, la température est située autour de $35^{\circ} \mathrm{C}$. La végétation est dominée par la steppe sahélienne à épineux à dominance Acacia seyal, Balanites aegyptiaca, Capparis spp., Combretum aculeatum et Ziziphus abyssinica (Fondoun, 2001; Jiagho et al., 2016). 


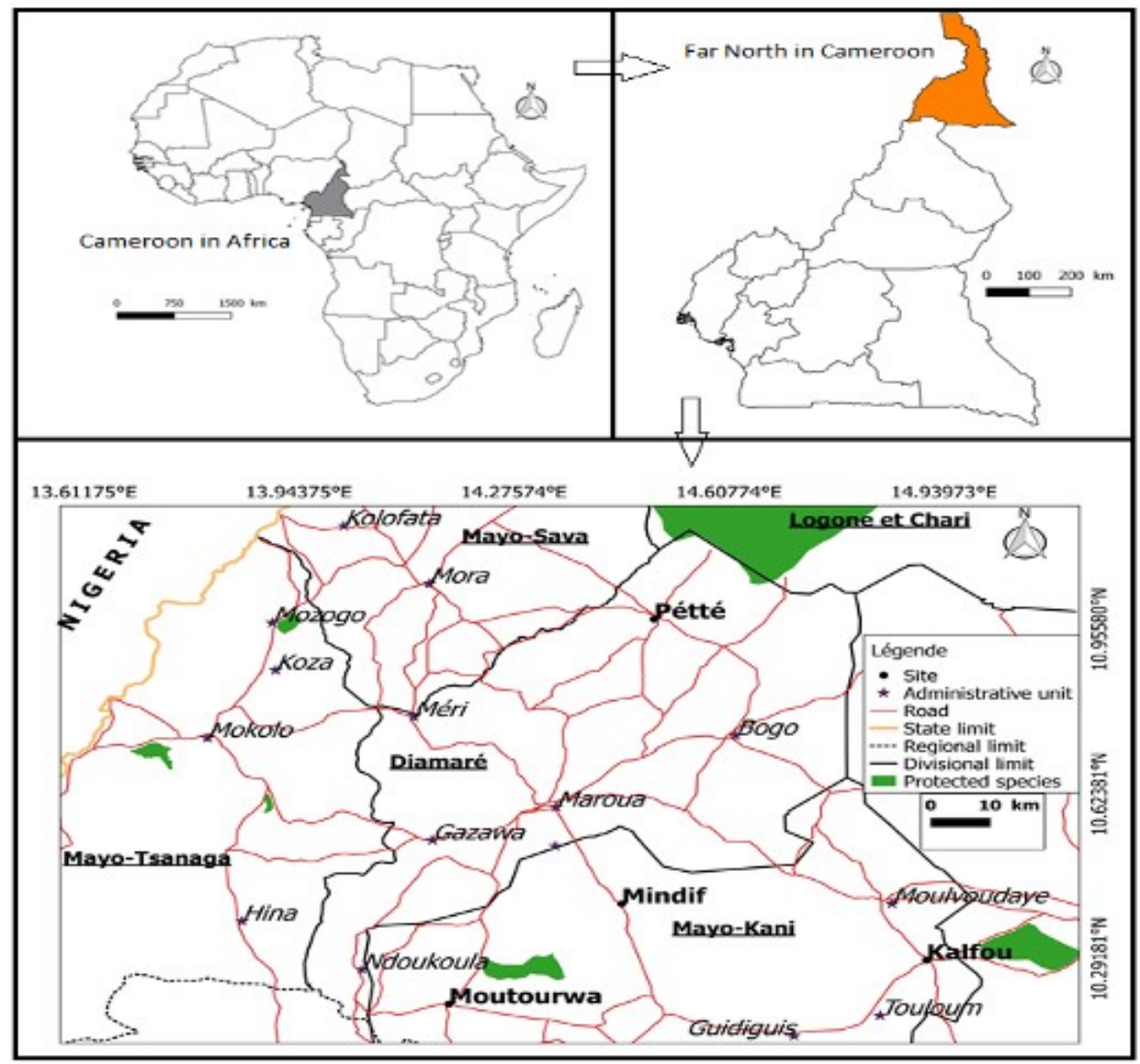

Figure 1: Carte de localisation des sites d'étude

Description de Haematostaphis barteri Hook. F.: Haematostaphis barteri est une espèce commune et disséminée, trouvée exclusivement en Afrique tropicale, de la Côte d'Ivoire au Soudan et partout au Ghana, Togo, Bénin, Cameroun et le Tchad. II est trouvé dans les terres boisées, spécialement sur les montagnes rocheuses et les éboulements dans les zones soudaniennes et guinéenne (Arbonnier, 2002). II est exclusivement présent dans les zones soudanosahéliennes. Haematostaphis barteri est un petit arbre ou arbuste de 2,0- 8,0 $\mathrm{m}$ de hauteur et avec une circonférence du tronc qui excède rarement $65 \mathrm{~cm}$. Les feuilles sont composées, glabres, alternées, et imparipennées. $H$. barteri est une espèce diö̈que, en raison de l'existence d'un pied mâle et d'un pied femelle. La floraison et la fructification commencent en fin de saison sèche. Les fruits sont des drupes ellipsoïdes, glabres, pourpres à maturité, lesquels sont portés par de longues panicules pendantes (Figure 2). Ils contiennent un noyau intégré dans une mince pulpe, comestible au goût acide. 


\section{Ittp://www.m.elewa.}

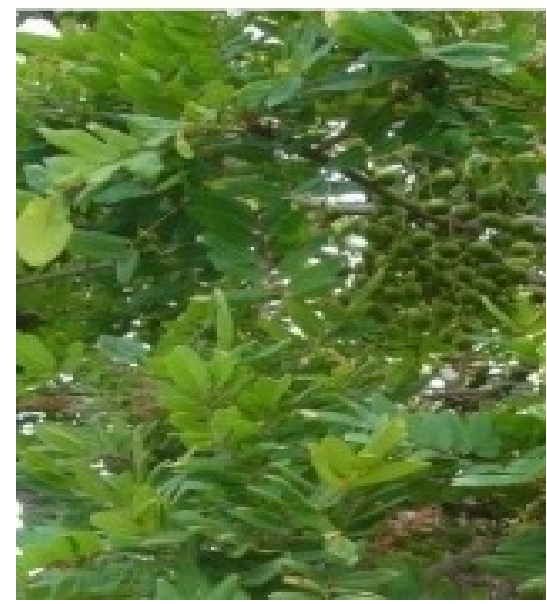

Figure 2 : Pied de Haematostaphis barteri portant une panicule pendante

Échantillonnage et collecte des données: Des parcelles carrées de 1 ha $(100 \mathrm{~m} \times 100 \mathrm{~m})$ ont été installées dans la savane. Un nombre total de 48 parcelles était conduit dans quatre sites présentés plus haut à raison de 12 parcelles par site. Dans chaque parcelle, tous les individus de Haematostaphis barteri ont été inventoriés. Les jeunes plantes comprenant des rejets de souches (individus régénérés après la coupe d'une tige) et libres ( $1 \mathrm{~cm} \leq \mathrm{dhp}<10 \mathrm{~cm}$ et hauteur $<1,5$ $\mathrm{m})$ ont été traitées des tiges d'avenir puis sont notées. Compte tenu de leur rareté, les semis étaient notés dans chaque parcelle d'inventaire de toute la zone. Les paramètres dendrométriques incluant le diamètre à hauteur de poitrine (dhp), la hauteur totale $(\mathrm{Ht})$, la surface terrière, le diamètre moyen ont été mesurés pour tous les individus de Haematostaphis barteri avec $\mathrm{dhp} \geq 10 \mathrm{~cm}$. L'évaluation de la production en fruits de $H$. barteri a été réalisée en 2015 sur des populations de la zone sahélienne du Cameroun, localisées à Moutourwa, Mindif, Kalfou et Pété. Elles ont été choisies en fonction de leur accessibilité. Au total, cette évaluation a porté sur un échantillon de 120 individus pris au hasard parmi les arbres rencontrés dans les parcelles délimitées précédemment à raison de 30 arbres par population considérée (Moutourwa, Mindif, Kalfou et Pété). Le choix a porté sur des individus qui n'avaient encore jamais été exploités, donc exempts de mutilation, et qui se trouvaient en pleine production des fruits. Les caractéristiques étudiées ont portées sur le nombre de panicule par arbre, le nombre de fruit par panicule et le nombre de fruit par arbre échantillonné. Ensuite, les relations avec certaines caractéristiques dendrométriques (dhp et $\mathrm{Ht}$ ) de l'arbre ont été établies.

Analyse des données

Caractérisation de la population de $\boldsymbol{H}$. barteri : Pour déterminer les caractéristiques dendrométriques de Haematostaphis barteri, les paramètres dendrométriques ont été calculés. Ces paramètres sont présentés dans le tableau 1. La caractérisation de la structure de Haematostaphis barteri est faite en utilisant les classes de distribution de diamètre et de hauteur.

Tableau 1 : Paramètres dendrométriques et leur formule

\begin{tabular}{l|r}
\hline Paramètres & Formules \\
\hline $\begin{array}{l}\text { Densité de la station (individus/ha) } \\
\text { Surface terrière }\left(\mathrm{m}^{2} / \text { ha) }\right. \\
\text { Diamètre moyen quadratique }(\mathrm{cm})\end{array}$ & $\mathrm{N}=$ Nombre total des individus par ha \\
$\mathrm{G}=\Sigma \pi \mathrm{D}^{2} / 4$ \\
$\begin{array}{l}\mathrm{D}_{\mathrm{g}}=\sqrt{\frac{4 G}{N \pi}} \\
\text { Indice de Blackman (Blackman, 1942) }\end{array}$ \\
$\begin{array}{l}\mathrm{IB}=\sigma^{2} / \mu ; \mathrm{IB}=1 \text { (distribution poissonnière) ; IB <1 (distribution } \\
\text { régulière) et } I \mathrm{~B}>1 \text { (distribution agrégée ou en bouquet) }\end{array}$ \\
\hline
\end{tabular}


$\mathrm{N}=$ densité de la population par hectare, $\mathrm{D}=$ diamètre de l'arbre, $\sigma^{2}=$ variance de la population d'arbres, $\mu=$ moyenne de la population d'arbre.

Selon Lykke (1998), la pente de régression et le coefficient de détermination étaient calculés pour chaque localité en ce qui concerne la distribution par classe de diamètre. La pente de distribution de la taille de classe résume en une seule valeur l'allure de la distribution de la taille des classes (Tabuti et Mugula, 2007). Si la population a une forte pente négative, elle est interprétée comme une population stable et naturellement capable de se remplacer elle-même. Par contre, les faibles pentes négatives ou les pentes plates montrent une faibles restauration et une population en déclin (Hall et Bawa, 1993 ; Lykke, 1998). Pour calculer la pente, le milieu de la taille de classe $\left(d_{1}\right)$ est considéré comme la variable indépendante et le nombre d'individus $\left(\mathrm{N}_{1}\right)$ de chaque classe comme variable dépendante. Pour avoir la droite, $\mathrm{N}_{1}$ est transformé en Ln $\left(\mathrm{N}_{1}+1\right)$ parce que certaines classes

\section{RESUTATS}

Distribution des classes de diamètre des arbres de Haematostaphis barteri: La figure 2 illustre les distributions de classe de diamètre des individus de Haematostaphis barteri pour les différents sites. L'analyse de la figure montre que les individus appartenant à la classe de diamètre inférieure à $20 \mathrm{~cm}$ sont plus nombreux quelle que soit le site. Les adultes sont peu représentés. Les populations de différents sites ont une faible distribution de classe de diamètre. Les pentes de régression et les coefficients de détermination sont respectivement : $a_{1}=-9,8 ; R_{1}{ }^{2}=0,95$ (Moutouroua); $a_{2}=-10,65 ; R_{1}{ }^{2}=0,93$ (Mindif); $a_{3}=-8,6$; $\mathrm{R}_{3}{ }^{2}=0,99$ (Kalfou) et $\mathrm{a}_{4}=-5,71 ; \mathrm{R}_{4}{ }^{2}=0,92$ (Pété). La distribution en fonction de classe de diamètre montre que les différentes populations sont faites essentiellement des populations juvéniles et qu'il y a n'ont aucun individu. La régression est calculée entre $\mathrm{d}_{1}$ et $\operatorname{Ln}\left(\mathrm{N}_{1}+1\right)$ (Lykke, 1998 ; Obiri et al., 2002).

Production de fruits de Haematostaphis barteri : Le nombre total de fruits par arbre, le nombre moyen de fruits par branche, le nombre moyen de fruits par panicule et le nombre de panicule par branche ont été déterminés. Une analyse de variance a été réalisée en utilisant le logiciel XLSTAT pour tester la différence statistique entre populations provenant de différents sites pour certaines variables telles que: nombre moyen de panicules par branche, nombre moyen de fruits par panicule et nombre moyen de fruit par arbre. Des tests de corrélation ont également été effectués entre les données dendrométriques et les productions fruitières.

une absence remarquables des individus adultes. Dans tous les sites, la distribution présente une forme en $\mathrm{J}$ renversé. Une telle forme met en relief que la population de $H$. barteri régénère bien dans la zone sahélienne mais que les individus adultes sont peu représentés et ne se maintiennent pas. La mortalité des jeunes pousses est très élevée dans tous les sites d'étude: 81,$13 ; \quad 89,28 ; \quad 73,33 ; \quad 65,57 \%$ respectivement à Moutourwa, Mindif, Kalfou et Pété (Figure 3). Les résultats de l'inventaire quantitatif ont révélé également que les différentes populations de cette Anacardiaceae ont été estimées à 497 individus/ha dont plus de 1/3 soit 189 individus/ha sont jeunes avec moins de $15 \mathrm{~cm}$ de dhp. Ces résultats traduisent la surexploitation de cette espèce par les communautés locales de cette région. 


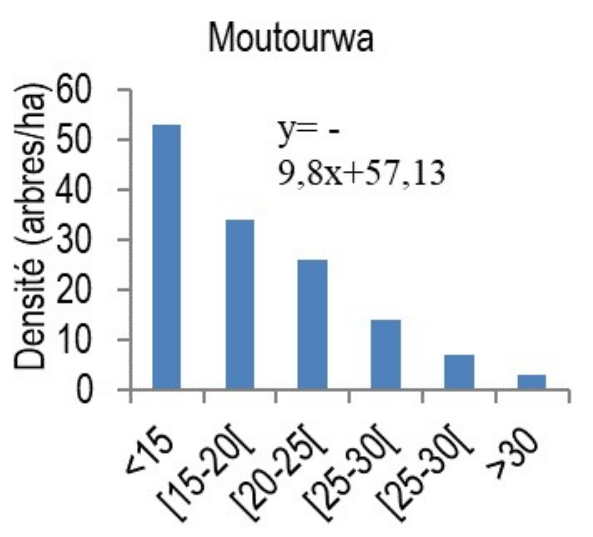

Classe de diamètre $(\mathrm{cm})$ Kalfou

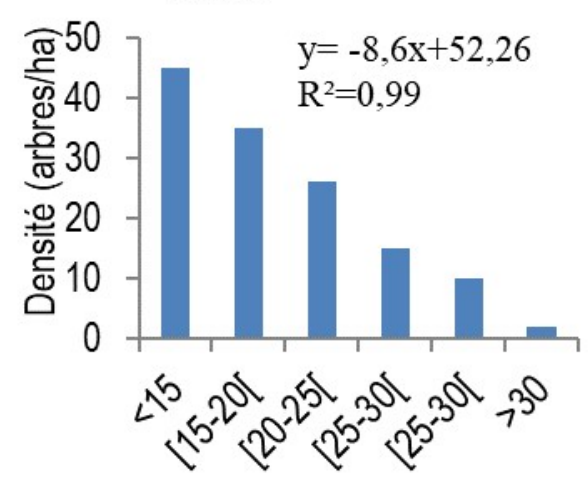

Classe de diamètre $(\mathrm{cm})$
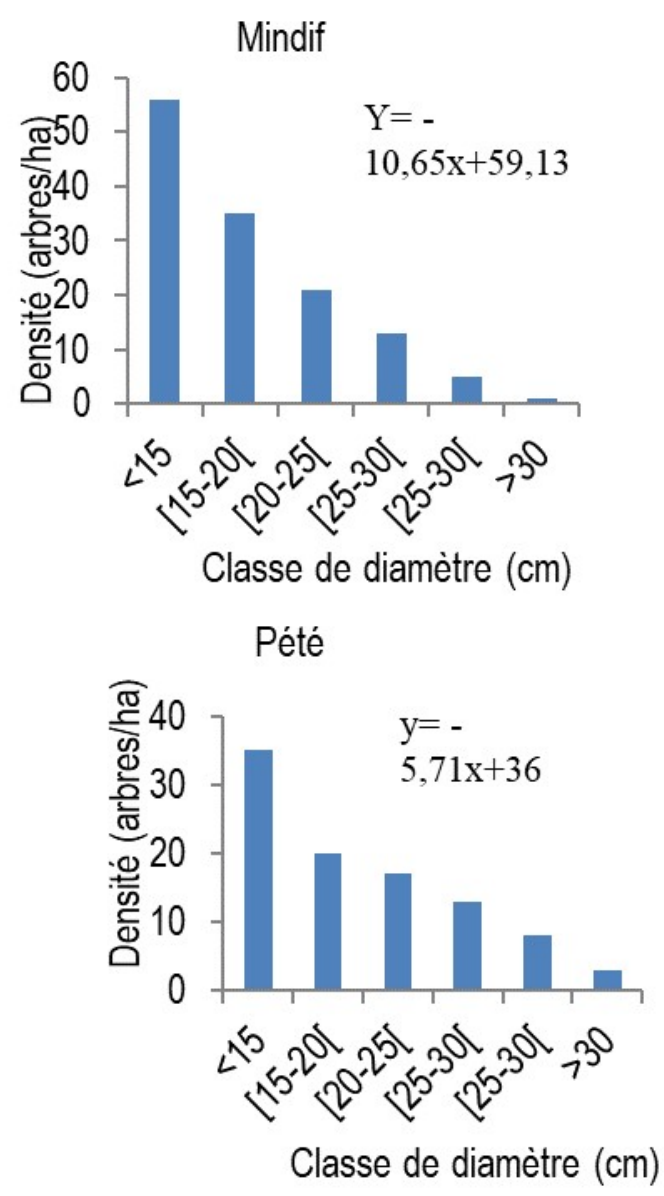

Figure 3 : Distribution de classes de diamètre de différentes populations de Haematostaphis barteri

Caractéristiques structurales des populations de Haematostaphis barteri: Le tableau 2 présente les caractéristiques structurales des populations de Haemotostaphis barteri. La densité varie de 13 individus/ha (Moutourwa) à 17 individus/ha (Mindif) tandis que la surface terrière s'étend de $0,39 \mathrm{~m}^{2} / \mathrm{ha}$ (Pété) à $0,49 \mathrm{~m}^{2} / \mathrm{ha}$ (Mindif). Le diamètre moyen quadratique varie de $17,31 \mathrm{~cm}$ (Moutourwa) à $20,57 \mathrm{~cm}$ (Pété). Le diamètre à hauteur de poitrine (dhp) et la hauteur totale sont respectivement de $16,16 \mathrm{~cm}$ et 4,75 $\mathrm{m}$ (Moutourwa), $18,10 \mathrm{~cm}$ et 5,00 $\mathrm{m}$ (Mindif), de 17,34 $\mathrm{cm}$ et $4,99 \mathrm{~m}$ (Kalfou), et $16,17 \mathrm{~cm}$ et $4,80 \mathrm{~m}$ (Pété). Les plus gros individus $(18,10 \mathrm{~cm})$ et les plus hauts $(5,00 \mathrm{~m})$ sont observés à Mindif tandis les plus minces individus $(16,16 \mathrm{~cm})$ et les plus courts $(4,75 \mathrm{~m})$ sont notés dans la population de Moutourwa. Le dhp, la hauteur, la surface terrière, la densité et le diamètre moyen montrent des différences significatives entre les populations $(P<0,0001)$.

Tableau 2: Caractéristiques structurales des populations de Haematostaphis barteri

\begin{tabular}{l|l|l|l|l|l|l}
\hline \multirow{2}{*}{ Localités } & \multicolumn{6}{|c}{ Caractéristiques structurales } \\
\cline { 2 - 7 } & dhp & Ht & ST & D & Dm & IB \\
\hline Moutrourwa & $16,16 \pm 0,41^{\mathrm{a}}$ & $4,75 \pm 0,12^{\mathrm{a}}$ & $0,28 \pm 0,03^{\mathrm{a}}$ & $13 \pm 2,63^{\mathrm{a}}$ & $17,31 \pm 2,60^{\mathrm{a}}$ & 0,55 \\
Mindif & $18,11 \pm 0,20^{\mathrm{c}}$ & $5,00 \pm 0,13^{\mathrm{b}}$ & $0,49 \pm 0,02^{\mathrm{c}}$ & $17 \pm 4,50^{\mathrm{c}}$ & $19,69 \pm 2,80^{\mathrm{c}}$ & 1,19 \\
Kalfou & $17,34 \pm 0,46^{\mathrm{b}}$ & $4,99 \pm 0,06^{\mathrm{c}}$ & $0,40 \pm 0,02^{\mathrm{b}}$ & $15 \pm 4,05^{\mathrm{b}}$ & $18,81 \pm 2,95^{\mathrm{b}}$ & 1,08 \\
Pété & $16,17 \pm 0,44^{\mathrm{a}}$ & $4,80 \pm 0,20^{\mathrm{a}}$ & $0,39 \pm 0,04^{\mathrm{b}}$ & $13 \pm 3,24^{\mathrm{a}}$ & $20,57 \pm 3,00^{\mathrm{d}}$ & 0,83 \\
Probabilité & $* * *$ & ${ }_{* * *}^{* * *}$ & ${ }_{* * *}$ & ${ }_{* * *}$ & \\
\hline
\end{tabular}

Les valeurs suivies d'une même lettre dans une colonne ne sont pas significativement différentes. dhp=diamètre à hauteur de poitrine ; Ht=Hauteur totale ; ST= Surface terrière ; $D=$ Densité ; $D m=$ Diamètre moyen ; IB= Indice de Blackmann. 
L'indice de Blackmann montre de diverses valeurs calculées. Cet indice est supérieur 1 à Kalfou $(1,08)$ et à Mindif $(1,19)$ indiquant que les individus de Haematostaphis barteri ont une distribution agrégative dans ces sites. Cette distribution apparaît irréaliste pour une densité de 15 à 17 individus/ha rencontrée dans ces sites même si dans certains endroits où les quadras ont été établis, des individus en agrégat ont été observés de manière éparse. Par contre, à Moutourwa $(0,55)$ et Pété $(0,83)$, l'indice de Blackmann montre des valeurs inférieures à 1 suggérant une distribution régulière. Dans ces sites, cette distribution paraît être raisonnable puisqu'elle montre l'importance de l'intervention de l'homme sur cette espèce surtout en ce qui concerne sa dispersion.

Rendement en fruits: Le tableau 3 présente les caractéristiques de la production fruitière de Haematostaphis barteri. Le nombre moyen de panicule par branche est d'environ 3 panicules à Mindif et 4 pour les autres sites. Le nombre moyen de fruits par panicule varie de 15 fruits (Mindif) à environ 28 (Kalfou). A Pété et à Moutourwa, le nombre moyen de fruits par panicule est d'environ 22 et 23 fruits respectivement. Le nombre moyen de fruits par arbre varie de 2345 (Mindif) à 7877 fruits (Moutourwa). A Pété et à Kalfou, le nombre de fruits par arbre est respectivement de 6092 et 6663 fruits.

Tableau 3 : Caractéristiques de la production fruitière de différentes populations de Haemtostaphis barteri

\begin{tabular}{l|c|c|c}
\hline Localités & $\begin{array}{c}\text { Nombre de panicule par } \\
\text { branches }\end{array}$ & $\begin{array}{c}\text { Nombre de fruits par } \\
\text { panicule }\end{array}$ & $\begin{array}{c}\text { Nombre de fruits par } \\
\text { arbre }\end{array}$ \\
\hline Moutourwa & $4,26 \pm 0,098^{\mathrm{c}}$ & $22,68 \pm 0,14^{\mathrm{c}}$ & $7877 \pm 7,25^{\mathrm{d}}$ \\
Mindif & $2,83 \pm 0,10^{\mathrm{a}}$ & $14,70 \pm 0,10^{\mathrm{a}}$ & $2346 \pm 11^{\mathrm{a}}$ \\
Kalfou & $3,89 \pm 0,042^{\mathrm{b}}$ & $27,66 \pm 0,053^{\mathrm{d}}$ & $6663 \pm 23,97^{\mathrm{c}}$ \\
Pété & $3,89 \pm 0,06^{\mathrm{b}}$ & $21,69 \pm 0,05^{\mathrm{b}}$ & $6092,53 \pm 97,04^{\mathrm{b}}$ \\
Moyenne & $3,66 \pm 0,55$ & $21,21 \pm 4,67$ & $5510,51 \pm 2095,36$ \\
Probabilité & $0,0001^{* * *}$ & $0,0001^{* * *}$ & $0,0001^{* * *}$ \\
\hline
\end{tabular}

Les valeurs suivies d'une même lettre dans une colonne ne sont pas significativement différentes

Que ce soit le nombre moyen de fruits par arbre, le nombre de panicule par branche et celui de fruits par panicule, l'analyse de variance montre une différence significative entre les différentes populations $(P<0,0001)$ (Tableau 3). Les branches avec le plus grand nombre de panicules sont rencontrées à Moutourwa alors que celles avec le plus petit nombre de panicules sont observées à Mindif.

Corrélation: Les résultats de l'analyse de corrélation entre le dbh, la hauteur totale et les variables de la production fruitière de Haematostaphis barteri sont présentés dans le tableau 4. Le tableau montre que le dbh est hautement corrélé avec le nombre de fruits par arbre $(r=0,893)$ et le nombre de panicule par branche $(r=0,611)$ tandis que la hauteur totale est significativement corrélée au nombre de fruits par arbre $(r=0,767)$. Cependant, le dbh et la hauteur totale ne sont pas corrélés avec le nombre de fruits par panicule. En plus, il y a une haute corrélation entre le dbh et la hauteur totale, entre le nombre de fruits par arbre et le nombre de panicule par branche et le nombre de fruits par panicule. II y a aussi une haute corrélation entre le nombre de panicule par branche et le nombre de fruits par panicule.

Tableau 4: Corrélation entre le dhp, la hauteur totale et les caractéristiques de la production fruitière

\begin{tabular}{l|c|c|c|c|c}
\hline Variables & dhp $(\mathbf{c m})$ & $\mathbf{H t}(\mathbf{m})$ & NF/Ar & NP/br & NF/P \\
\hline $\mathrm{dhp}(\mathrm{cm})$ & 1 & & & & \\
$\mathrm{Ht}(\mathrm{m})$ & $\mathbf{0 , 6 9 5}$ & 1 & & & \\
$\mathrm{NF} / \mathrm{Ar}$ & $\mathbf{0 , 8 9 3}$ & $\mathbf{0 , 7 6 7}$ & 1 & & \\
$\mathrm{NP} / \mathrm{br}$ & $\mathbf{0 , 6 1 1}$ & 0,095 & $\mathbf{0 , 6 5 0}$ & 1 & \\
$\mathrm{NF} / \mathrm{P}$ & 0,087 & 0,140 & $\mathbf{0 , 6 6 1}$ & $\mathbf{0 , 7 8 9}$ & 1 \\
\hline
\end{tabular}

Les valeurs en gras sont significativement différentes de 0 à un niveau de signification alpha=0,05; dhp=Diamètre à hauteur de poitrine ; $\mathrm{Ht}=$ Hauteur totale ; NF/Ar= Nombre de fruits par arbre ; NP/br= Nombre de panicule par branche ; NF/P= Nombre de fruit par panicule. 
Régression: Le tableau 5 présente les équations de régression linéaire entre les paramètres dendrométriques et certains descripteurs de la production fruitière. Ces équations de régressions calculées à partir de ces mêmes descripteurs de la production fruitière de $\mathrm{H}$. barteri montrent qu'il existe une régression significative entre le nombre de panicules par branche et le dbh, entre le nombre de fruit par arbre et le dbh d'une part et le nombre de panicule par branche et la hauteur totale de l'arbre d'autre part. Par contre, la régression n'est pas significative entre le nombre de fruits par arbre et la hauteur totale de l'arbre.

Tableau 5 : Régression linéaire entre le dbh, la hauteur totale et certains descripteurs de production des fruits (NP/br, NF/Ar)

\begin{tabular}{l|c|c|c}
\hline Équation de la régression & $\mathbf{R}^{\mathbf{2}}$ & Probabilité & Signification \\
\hline $\mathrm{NP} / \mathrm{br}=-0,65+0,27^{*} \mathrm{dbh}(\mathrm{cm})$ & 0,483 & 0,0001 & $* * *$ \\
$\mathrm{NF} / \mathrm{Ar}=-6484,59+790,23^{*} \mathrm{dbh}(\mathrm{cm})$ & 0,799 & 0,0001 & $* * *$ \\
$\mathrm{NP} / \mathrm{br}=3,29+0,14^{*} \mathrm{Ht}(\mathrm{m})$ & 0,067 & 0,0001 & $* * *$ \\
$\mathrm{NF} / \mathrm{Ar}=6828,41+34,73^{*} \mathrm{Ht}(\mathrm{m})$ & 0,001 & 0,593 & $\mathrm{~ns}$ \\
\hline
\end{tabular}

$\mathrm{R}^{2}=$ Coefficient de détermination ; dhp=Diamètre à hauteur de poitrine ; $\mathrm{Ht}=$ Hauteur totale ; NF/Ar= Nombre de fruits par arbre ; $\mathrm{NP} / \mathrm{br}=$ Nombre de panicule par branche ; NF/P= Nombre de fruit par panicule.

Les droites de régression (Figures 4) indiquent un coefficient de détermination faible pour les régressions linéaires entre le nombre de panicule par branche et le dhp d'une part et le nombre de panicule par branche et

\section{DISCUSSION}

Caractéristiques structurales des populations de Haematostaphis barteri : La hauteur totale moyenne et le dhp ont montré de différence significative au seuil de 0,0001 (Tableau 2). La population de Mindif distribuée dans la zone la plus anthropisée présente de la hauteur totale d'autre part. Par contre, le coefficient de détermination est élevé pour la régression linéaire entre le nombre de fruit par arbre et le dhp. Dans les deux cas, les régressions sont hautement significatives.

plus en plus de hauts arbres que ceux des autres populations. De même, la population de Mindif montre les valeurs les plus élevées en ce qui concerne les autres paramètres structuraux tels que la densité, la surface terrière (Tableau 2). 

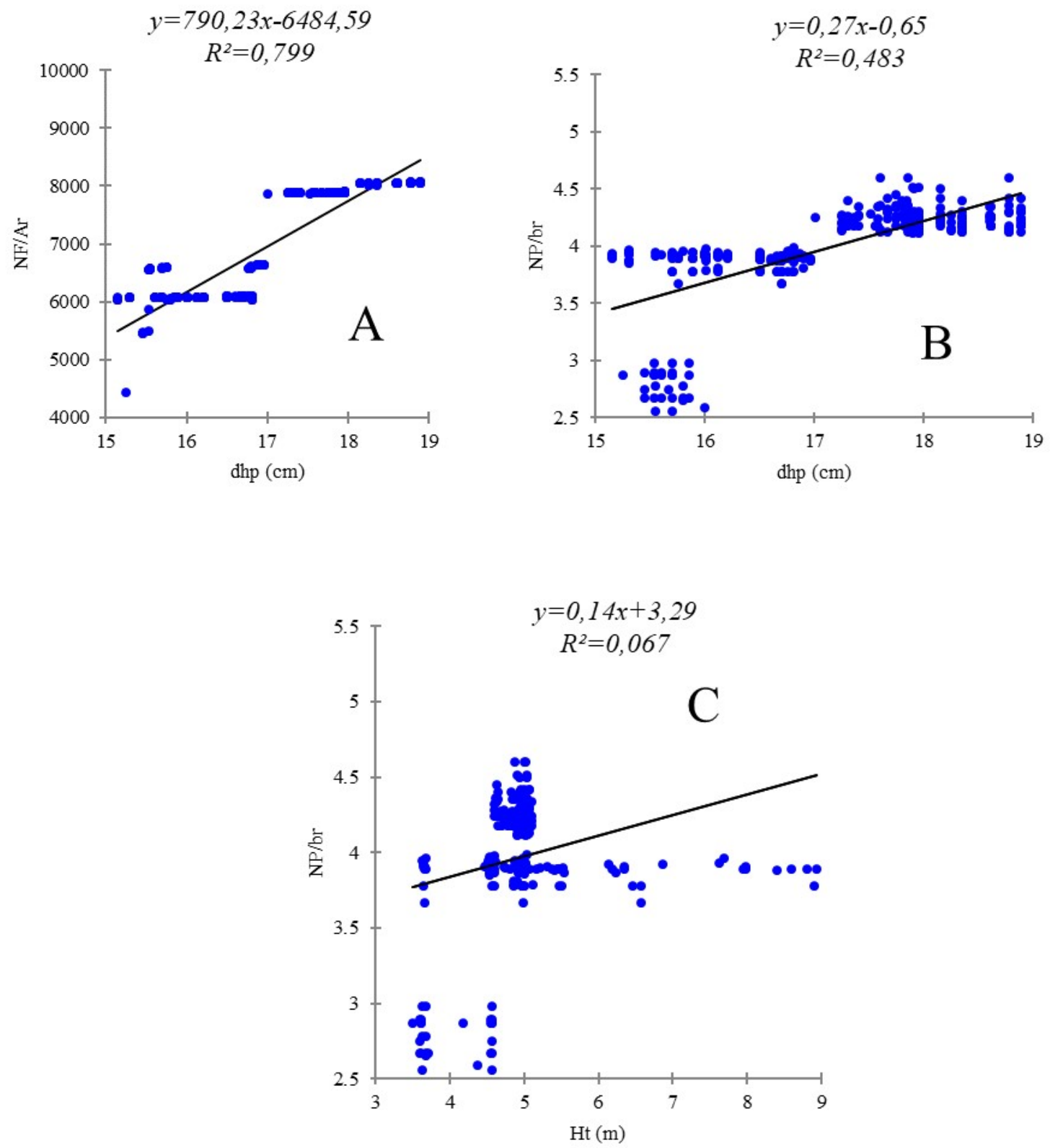

Figure 4 : Relation entre le nombre de fruits par arbre et le dhp $(A)$, entre le nombre panicule par branche et le dhp (B) et entre le nombre de panicule par branche et la hauteur totale (C) de $\mathrm{H}$. barteri

Cela indique que les communautés locales de cette région contribuent énormément à la conservation des ressources naturelles. De plus les activités menées par certaines organisations non gouvernementales telles que l'Organisation Néerlandaise de Développement (SNV) ont aidé la communauté locale à prendre conscience de la nécessité de la gestion durable des ressources. Par ailleurs, le sol du site de Mindif est quelque peu rocailleux; ce qui améliorait le développement des $H$. barteri à Mindif comme l'ont soulevé Sourou et al., (2016). Les milieux à sols rocailleux constitueraient l'habitat préféré de Haematostaphis barteri. Ce constat corrobore les résultats des travaux sur $H$. barteri dans les zones sèches d'Afrique de l'Ouest (Arbonnier, 2005) et le nord du Togo (Eyana, 2007 ; Agbogan et al., 2012). La distribution diamétrale de la population de Haematostaphis barteri dans les quatre sites a 
clairement révélé la prédominance de jeunes individus ou petits diamètres mais elle ne peut pas être interprétée comme une bonne conservation des espèces ou que l'espèce régénère bien. L'analyse de cette distribution des classes de diamètre indique que l'espèce a une faible structure de la population exhibant une allure en $\mathrm{J}$ renversé (Figure 2). Généralement une espèce à faible pente négative a une pauvre régénération et peut disparaître (Tabuti et Mugula, 2007). La distribution chez $H$. barteri présente de forte pente négative indiquant un rétablissement de l'espèce. Malheureusement, les jeunes tiges ne parviennent pas à la maturité à cause d'une pression anthropique sur celles-ci. La prédominance des jeunes individus serait liée non seulement à la physiologie de l'espèce, qui est un arbuste dont le diamètre dépasse rarement $35 \mathrm{~cm}$ (Agbogan et al., 2012), mais aussi sa perturbation ou sa vulnérabilité à certaines étapes de son développement (Ouédraogo et al., 2006). Le développement des individus jeunes est perturbé par leur disparition due à l'exploitation abusive de l'espèce ou au surpâturage. Ce qui affaiblirait ainsi la population. Pour se maintenir, la population a besoin d'un nombre important de jeunes individus qui pourront être intégrés dans la classe des adultes (Batiano et al., 2001). L'absence des individus adultes ou semenciers dans la population affecterait également le rétablissement de la population par le manque des graines (Mapongmetsem et al., 2011). Le taux de mortalité élevé des jeunes individus s'explique par la constante perturbation de la surexploitation. Malgré le fait que cette distribution montre une allure identique dans tous les sites, il est important de signaler que la structure de l'espèce varie selon le milieu. Ce constat a été fait par Durrieu et al., (1997) qui ont montré que la structure d'une espèce peut varier à l'échelle d'une station ou à travers toute une région. Par exemple, au Ghana, Hawthorne (1995) a montré que la majorité des espèces forestières ont une régénération naturelle suffisante et la plupart de ces espèces montrent une distribution en forme de "J renversé". Cette forme de distribution obtenue avec les classes de diamètre de $H$. barteri corrobore bien avec les résultats obtenus au Bénin par Glèlè kakaï et Sinsin (2009) et Natta et al. (2011) respectivement sur les populations de Pentadesma butyracea et Isoberlinia spp. Des résultats similaires ont également été obtenus par Yehouenou Tessi et al. (2012), Dicko et al. (2017) respectivement avec Antiaris toxicaria et Lophira lanceolata. Cependant, cette structure ne peut pas seulement être dérivée du comportement de l'espèce mais aussi reliée à la pression anthropique.
Production fruitière et gestion durable de la population de Haematostaphis barteri populations dans la zone sahélienne du Cameroun: Même comme le nombre moyen de fruits par arbre ne varie pas significativement d'une population à l'autre, les arbres de la population de Moutourwa qui sont significativement moins larges et élevés que les autres populations sont les plus productifs en nombre de fruits (7877 fruits par arbre). Cependant, les arbres de la population de Mindif (2346 fruits par arbre) sont moins productifs, bien qu'ils soient significativement plus larges et plus élevés que les autres. Ceci suggère que l'environnement influence significativement le développement végétatif de Haematostaphis barteri et sa production en termes de nombre moyen de fruits par arbre (Boffa, 2000 ; Lamien et al., 2003). Le rendement moyen en fruits de Haematostaphis barteri obtenu est significativement supérieur à celui obtenu par Dan Guimbo et al. (2012) ; Sanogo et al. (2014) et Kouayaté et al. (2016) respectivement sur les arbres de Adansonia digitata, Parkia biglobosa et Vitellaria paradoxa. Cette différence serait en partie liée à la physiologie de l'espèce et aussi aux différentes méthodes de quantification utilisées. Alors que la méthode de quantification des fruits de Adansonia digitata et Vitellaria paradoxa utilisée par Dan Guimbo et al. (2012) et Kouyaté et al. (2016) était basée sur le dénombrement systématique de tous les fruits, celle utilisée pour la quantification de la production de Haematostaphis barteri est basée sur un échantillonnage aléatoire des principales branches (Sourou et al., 2016a). La corrélation significative et positive entre le diamètre du tronc et le nombre de fruit par arbre et le nombre de panicule par branche d'une part et la hauteur et le nombre de panicule par branche d'autre part de $H$. barteri, corroborent es résultats d'autres travaux réalisés sur des espèces fruitières forestières soudano-sahéliennes (Kouyaté et al., 2006, Sanogo et al., 2014 ; Dan Guimbo et al., 2012). Ces corrélations permettent de dire que dans la population de $H$. barteri, les arbres bons producteurs sont de gros diamètre. Ce constat s'accorde avec les conclusions de Boffa (2000) et Lamien et al. (2004) qui soutiennent que le nombre et le poids moyen des fruits de Vitellaria paradoxa étaient positivement corrélés à la taille de l'arbre. Par contre, au Burkina Faso, Guira et Zongo (2002) ont trouvé une corrélation négative entre le diamètre et la production de Vitellaria paradoxa indiquant que Chez cette espèce, les arbres bons producteurs sont de petite taille. Les différentes populations sont en état de vulnérabilité en raison de 
leur vieillissement dû à la rareté de la régénération naturelle et accentuées non seulement par la prédation des graines par les petits rongeurs mais aussi par la forte pression. En effet, les différentes parties de l'espèce sont utilisées. Cette situation a également été soulignée par Sourou et al. (2016a) et Biaou et al. (2017) au Togo. Comme les principaux organes de l'espèce sont d'une grande importance (Gaoué et Ticktin, 2007; Hawkes et Sullivan, 2010; Allred et al., 2012), la gestion durable des populations de Haematostaphis barteri est une nécessité pour améliorer les moyens de subsistance de la communauté locale. Une sensibilisation efficace de ces communautés locales pour la protection des espèces

\section{CONCLUSION}

L'étude des structures diamétriques de différentes populations de Haematostaphis barteri montre que la régénération naturelle de l'espèce est bonne dans la zone d'étude avec des individus jeunes plus nombreux que des vieux. Cependant, ils ne parviennent pas toujours à la maturité car ils sont très vulnérables face aux actions humaines et à des facteurs naturels. Ainsi, les équations de régression présentent de fortes pentes négatives montrant la dominance des individus jeunes et une absence remarquable des individus adultes. L'évaluation de la production en fruits de $H$. barteri a permis de mettre en évidence l'existence d'un potentiel important. La production moyenne par arbre de $H$. barteri présente une grande variabilité entre les sites. Le rendement moyen de fruit par arbre ne montre de

\section{REMERCIEMENTS}

Les auteurs remercient l'Université de Maroua pour le financement d'une partie du travail. Ils remercient également les guides qui ont bien voulu les

\section{REFERENCES}

Adomou CA, 2005. Vegetation pattern and environmental gradients in Benin: Implication for biogeography and conservation. $\mathrm{PhD}$ thesis, Wageningen University; 2005.

Agbogan A, Tozo K, Wala K, Batawila K, Dourma M, Akpagana K. 2012. Abondance et structure des populations d'un fruitier spontané: Haematostaphis barteri Hook $\mathrm{F}$ dans deux sites rocheux en région soudanienne au Togo. Int. J. Biol. Chem. 6(6):6042-6048. DOI: 10.4314/ijbcs.v6i6.31

Allred BW, Fuhlendorf SD, Smeins FE, Taylor CA., 2012. Herbivore species and grazing intensity en jachère et en conservation in situ dans les savanes de la zone sahélienne du Cameroun pourrait permettre une conservation future. II est important de protéger la régénération naturelle contre les incendies et les coupes abusives de bois, mais aussi celles des porteurs de graines dispersées dans les champs. L'étude de la reproduction sexuée (mode de reproduction, dormance, etc.) en milieu naturel et en laboratoire et des essais de propagation végétative à faible coût (Bellefontaine et al., 2015) sont des études à entreprendre pour envisager un éventuel enrichissement des terres boisées par Haematostaphis barteri.

différence significative entre les différentes populations de $H$. barteri. Cependant la population de Mindif est la moins productive en termes de nombre moyen de fruit par arbre. Les équations de régression établies constituent des outils aptes à évaluer la production, voire la productivité des individus domestiqués de $H$. barteri. Des dispositions doivent être prises pour assurer le maintien des jeunes individus afin de ne pas compromettre, à terme, l'utilisation durable de l'espèce. Pour les buts de domestication et de conservation, des investigations doivent être faites particulièrement sur la caractérisation morphologique des fruits, le mode de reproduction et l'importance socioéconomique de Haematostaphis barteri.

accompagner sur le terrain ainsi que les lecteurs anonymes pour leurs commentaires qui ont été utiles pour améliorer de la qualité du manuscrit.

regulate community composition and an encroaching woody plant in semi-arid rangeland. Basic Appl. Ecol. 13(2):149-158.

Arbonnier M. 2002. Arbres arbustes et lianes des zones sèches d'Afrique de l'Ouest. CIRAD-MNHNUICN. 2002;573.

Bationo BA, Ouedraogo SJ, Guinko S, 2001. Longévité des graines et contraintes à la survie des plantules d'Afzelia africana Sm. dans une savane boisée du Burkina Faso. ANN. For. Sci., 58(1), 65-75.

Bellefontaine R., Meunier Q, Aboubacar I, Le Bouler H., 2015. Multiplication végétative à faible coût au 
profit des paysans et éleveurs des zones tropicales et méditerranéennes. VertigO - la revue électronique en sciences de l'environnement URL : http://journals.openedition.org/vertigo/16516

Biaou, SS. H., Moutouama, J. K., Dan, B. S. C., Amahowé, O. I., Moutouama, F. T., \& Natta, A. K. (2017). Uses of Haematostaphis barteri Hook.f. among the Waaba and Bètammaribè in North-Benin and impact on the species vulnerability. International Journal of Biodiversity and Conservation, 9(5), 146-157.

Boffa JM, 2000. West African Agroforestry parklands: keys to conservation and sustainable management, Unasylva English Ed. 51: 11-17.

Breman H, Kessler JJ, 1995. Woody plants in agroecosystems of semi-arid regions with emphasis on the Sahelian countries, SpringerVerlag, Adv. Ser. Agric. Sci. 23, Berlin, Germany, $340 \mathrm{p}$.

Dan Guimbo I, Larwanou M, Mahamane A, Ambouta KJ-M, 2012. Production fruitière de Neocarya macrophylla (Sabine) Prance, espèce ligneuse alimentaire du Niger. Journal of Applied Biosciences 60: 4388- 4393.

Donfack $P, 1998$. Végétation des jachères du NordCameroun: typologie, diversité, dynamique, production. Thèse de Doct. D'état. Univ. de Yaoundé 1. Cameroun.

Dicko A, Natta AK, Biaou HSS, Balagueman R, Gouwakinnou $G$ 2017. Typology and structural characterization of Lophira lanceolata populations in Benin (West Africa). Rev. Cames 5 (1): 35-43.

Durrieu De Madron L, Forni E, 1997. Aménagement forestier dans l'Est du Cameroun; Structure du peuplement et périodicité d'exploitation. Bois For. Trop. 1997; 254:39-49.

Eyana KA, 2007. Les Anacardiaceae du Togo: Études Botaniques, Écologiques et Propriétés antifongiques. Thèse de doctorat, Université de Reims, p.183.

Fondoun JM, 2001. Situation des ressources génétiques forestières du Nord Cameroun. Atelier sous-régional FAO/IPGRI/ICRAF sur la conservation, la gestion, l'utilisation durable et la mise en valeur des ressources génétiques forestières de la zone sahélienne (Ouagadougou, 22-24 sept. 1998). Note thématique sur les ressources génétiques forestières. Document FGR/15F. Département des forêts, FAO, Rome, Italie.

Gaoué OG, Ticktin T., 2007. Patterns of harvesting foliage and bark from the multipurpose tree Khaya senegalensis in Benin: variation across ecological regions and its impacts on population structure. Biological Conservation 137: 424-436.

Garnier A, 2006. Qu'est-ce qu'une espèce rare? Origines et fonctionnement de la rareté naturelle. Travail Bibliographique, DEA BEFA; 2008.

Gautier D, Hautdidier B, Ntoupka M, Onana J, Perrot N, Tapsou T, 2002. Fiches techniques des arbres utiles aux paysans du Nord Cameroun. Caractéristiques de l'arbre, ce qu'en font les paysans et ce qu'ils pourraient en faire. 2002. $<$ hal-00837556>.

Glèlè Kakaï $R$, Sinsin B, 2009. Structural description of two Isoberlinia dominated vegetation types in the Wari-Maro Forest Reserve (Benin). South African Journal of Botany, 75: 43-51.

Guira M et Zongo JD, 2002. Évaluation de la production d'une population de karité, Vitellaria paradoxa (Gaertn.f) (Sapotaceae) au Burkina Faso. Bulletin de la Recherche Agronomique 38 : 1625.

Hall P, Bawa, K, 1993. Methods to assess the impact of extraction of Non-Timber Tropical Forest Products on plant populations. Econ. Bot., 47, 234-247.

Hawkes CV, Sullivan JJ, 2010. The impact of herbivory on plants in different resource conditions: a meta-analysis. Ecology, 82(7):2045-2058.

Hawthorne WD, 1995. Ecological profiles of Ghanaian forest trees, Tropical Forest 29. Oxford Forestry Institute, Dept. of Plant Sciences, South Parks Rd, Oxford OX1 3RB, UK.

Jiagho E, Zapfack L, Banoho L, Tsayem-Demaze M., Corbonnois J, Tchawa P., 2016. Diversité de la flore ligneuse à la périphérie du Parc national de Waza (Cameroun). [VertigO] La revue électronique en sciences de l'environnement ,16(1).

Kouyaté AM, VanDamme P, Diawara H., 2006. Évaluation de la production en fruits de Detarium microcarpum Guill. \& Perr. Au Mali. Fruits, $61: 267-272$.

Lamien N, Ouédraogo SJ, Diallo OB, Guinko S., 2004. Productivité fruitière du karité (Vitellaria paradoxa Gaertn. C. F., Sapotaceae) dans les 
parcs agroforestiers traditionnels au Burkina Faso. Fruits, 59, 423-429.

Le Houerou HN., 1980. Techniques agroforestières pour la conservation et l'amélioration de la fertilité des sols dans les zones arides et semiarides, in : Le Houerou H.N. (Éd.), Les fourrages ligneux en Afrique. État actuel des connaissances, Colloq. int. fourrag. Ligneux Afr., CIPEA, Addis Ababa, Éthiopie, pp. 421424.

Lykke AM, 1998. Assessment of species composition change in savanna vegetation by means of woody plants size class distributions and local information. Biodiv. and Conserv., 7, 12611275.

Mapongmetsem PM, Nkongmeneck BA, Rongoumi G, Dongock DN, Dongmo B. 2011. Impact des systèmes d'utilisation des terres sur la conservation de Vitellaria paradoxa Gaerten. F. (Sapotaceae) dans la région des savanes soudano-guinéennes. Internat. J. Environ. Studies, 68 (6), 851-872.

Natta AK, Yédomonhan $\mathrm{H}$, Zoumarou Wallis $\mathrm{N}$, Houndéhin J, Ewédjè EBK, Glèlè Kakaï RL, 2011a. Typologie et structure des populations naturelles de Pentadesma butyracea dans la zone soudano-guinéenne du Bénin, Annales des Sciences Agronomiques, $15: 217-243$.

Natta AK, Adomou AC, Tchabi VI, Sogbegnon AR, Mensah GA, Sinsin BA, 2011b, Inventaire, typologie et structure des populations naturelles de Pentadesma butyracea (Clusiaceae) de la chaîne de l'Atacora au Nord-Ouest du Bénin, Bulletin de la Recherche Agronomique du Bénin, 70 : 10-24

Ngueguim JR, Zapfack L, Youmbi E, Riera B, Onana J, Foahom B, Makombu JG, 2010. Diversité floristique sous canopée en plantation forestière de Mangombe-Edea (Cameroun). Biotechnol. Agron. Soc. Environ. 14(1), 167176.

Obir J, Lawes M, Mukolwe, 2002. The dynamic and sustainable use of high-value tree species of the coastal Pondoland forest of the Eastern Cape Province, South Africa. Forest Ecol. and Manag., 166, 131-148.
Ouédraogo A, Thiombiano A, Hahn-Hadjali K, Guinko S. 2006. Diagnostic de l'état de dégradation des peuplements de quatre espèces ligneuses en zone soudanienne du Burkina Faso. Sécheresse, 17(4): 485-491. DOI: 10.1684/sec.2006.0058.

Sanogo D, Badji M, Diop M, Samb CO, Tamba A, Gassama YK., 2014. Évaluation de la production en fruits de peuplements naturels de Baobab (Adansonia digitata L.) dans deux zones climatiques au Sénégal. J. Appl. Biosci. 85:7838- 7847

Sourou BN, Ouinsavi C, Sokpon N., 2016a. Ecological Structure and Fruit Production of Blood Plum (Haematostaphis barteri Hook. F.) Subpopulations in Benin. International Journal of Plant \& Soil Science, 9(2): 1-12. DOI: 10.9734/IJPSS/2016/22059.

Sourou B N, Yabi J, Ouinsavi CAIN, et Sokpon N. 2016b. Importance socio-économique de la prune rouge (Haematostaphis barteri Hook F.) au Bénin. Int. J. Biol. Chem. Sci. 10(1): 326343.

Suchel JB, 1987. Rainfall patterns and regimes rainfall in Cameroon. Doc. Geographic tropical, No. 5, CEGET-CNRS, Talence, $287 \mathrm{p}$.

Tabuti JRS, Mugula BB, 2007. The ethnobotany and ecology status of Albizia coriaria Welw. ex Oliv. in Budondo Sub-country, eastern Uganda. Afr. J. Ecol., 45 (Supplement 3), 126129.

Wong JLG, Thornber K, Baker N, 2001. Evaluation des ressources en produits forestiers non ligneux: Expérience et Principes de Biométrie. FAO. 2001;52.

Yehouenou Tessi RD, Akouehou GS, Ganglo JC., 2012. Caractéristiques structurales et écologiques des populations de Antiaris toxicaria (Pers.) Lesch et de Ceiba pentandra (L.) Gaertn dans les forêts reliques du SudBenin. Int. J. Biol. Chem. Sci. 6(6): 5056-5067,

Yengue J-L, Callot $Y, 2002$. L'arbre et la ville dans la région de Maroua (Extrême-Nord Cameroun). Science et changements planétaires/Sécheresse, 13 (3):155-163. 